\title{
Discharge pattern of single motor units in the tonic vibration reflex of human triceps surae
}

\author{
DAVID BURKE ${ }^{1}$ A NDHANSH. SCHILLER² \\ From the Department of Clinical Neurophysiology, \\ Academic Hospital, Uppsala, Sweden
}

SYNOPS IS Using a single fibre EMG electrode the firing pattern of 46 motor units in the triceps surae has been studied during vibration of the Achilles tendon at frequencies of 25-200 Hz. Potentials activated in the tonic vibration reflex (TVR) were phase-locked to the vibration cycle but tended to become somewhat less so with continued vibration. The firing pattern of voluntarily activated motor units became locked to the waveform by the application of the vibrator. The discharges of 21 motor units were studied during low threshold (sub-M wave) tetanic stimulation of the tibial nerve at $25-100 \mathrm{~Hz}$. No evidence was found of synchronization of potentials activated in the resulting tonic contraction. During weak voluntary contractions, stimulation also failed to regularize voluntarily activated motor units. The findings can be reconciled by postulating that, in normal man, vibration activates monosynaptic and polysynaptic pathways, the latter circuit being adequate to generate reflex contraction, while the former merely affects the temporal patterning of the motor outflow.

The suggestion that the reflex pathways mediating the tonic vibration reflex (TVR) are, at least in part, polysynaptic has aroused little debate (cf. Kanda, 1972). The presence of the necessary polysynaptic excitatory group Ia pathways from the primary spindle ending, the receptor presumed responsible for transducing the vibratory stimulus, is less well documented than the classical monosynaptic pathway. Paradoxically, however, the role of the monosynaptic pathway in the TVR remains unclear.

In the cat, detailed analysis of the firing pattern of single motor units activated by vibration has established that the latencies between successive motoneurone discharges are always integer multiples of the duration of the vibration cycle (Homma et al., 1972a). This principle can be expressed as a 'decoding ratio', the ratio between vibration frequency

\footnotetext{
${ }^{1}$ C. J. Martin Travelling Fellow of the National Health and Medical Research Council of Australia.

${ }^{2}$ Fellow of the Schweizerische Stiftung für Medizinisch-Biologische Stipendien.

(Accepted 10 February 1976.)
}

and motoneurone discharge frequency (Homma et al., 1972a). Similar analyses in man of EMG activity induced by vibration of the quadriceps muscle at $100 \mathrm{~Hz}$ have suggested that 'the principle of integer multiplication' may also be applied to the human TVR (Homma et al., 1972b; Homma and Kanda, 1973; Hirayama et al., 1974). The finding that, with continued vibration, some motor unit discharges are not locked to the vibration wave whereas others are has been taken by Homma and colleagues to indicate that not only monosynaptic but also polysynaptic activation of the motoneurone occurs during the TVR.

Godaux and Desmedt (1975) observed that the TVR of the human masseter muscle is characterized by a distinct grouping of the gross EMG activity into bursts monosynaptically locked to the vibration frequency. They suggested that this vibration-induced timing of EMG activity indicates a greater role of the monosynaptic pathway in the TVR of the masseter than in the TVR of human limb muscles, and in recent papers they have shown 
that in the masseter the discharge of single motor units is locked to the vibration wave with a very small 'jitter' (Desmedt and Godaux, 1975), while in the limb muscles the latency 'jitter' for single motor units is larger (Godaux et al., 1975). In the masseter muscle the synchronization of EMG activity to the vibration wave has been confirmed by Hagbarth et al. (1976), who point out, however, that the reflex mechanisms responsible for the TVR contraction should be regarded separately from those responsible for the vibration-induced timing of motor discharges: any rhythmical input, facilitatory or inhibitory, will tend to synchronize motoneurone discharge to the input provided that the input is not dispersed in its afferent path. Hagbarth et al. (1976) attribute the marked EMG synchrony of the masseter TVR to the shortness of the reflex arc, resulting in little dispersion of neural volleys.

If dispersion of the afferent volley due to the length of the reflex arc is the factor responsible for the differences between the masseter TVR and the limb muscle TVR noted in gross multi-unit EMG recordings by Godaux and Desmedt (1975), it would be of interest to examine the firing pattern of single motor units activated during the TVR in muscles with a long reflex arc, such as triceps surae, and in a brief report published after the completion of the present study, this has been done (Godaux et al., 1975). With a relatively long reflex arc and with relatively high frequencies of vibration (150-200 Hz), the degree of dispersion of the afferent volley would result in continuous afferent bombardment of the motoneurone. The demonstration of motor unit discharges locked to the vibration waveform under such conditions could shed further light on the possible monosynaptic nature of the vibration-induced timing phenomenon. The present study was therefore undertaken to reassess in human triceps surae the relationship between vibration frequency and the activity of single motor units. Additionally, observations have been made on the tonic contraction induced by low threshold tetanic stimulation of muscle afferents, since previous studies (de Gail et al., 1966; Lang and Vallbo, 1967) have reported an absence of correlation be- tween the timing of motor unit discharge and the electrical stimulus, despite the less dispersed nature of the afferent input.

\section{METHODS}

Seven experiments were performed on six normal adult subjects. Experiments were performed with subjects lying on their left side on a comfortable bed, the right leg extended and the right foot fixed to the plate of a hydraulic device, details of which have been given previously (Hagbarth et al., 1975). Experiments were begun with the foot at right angle to the line of the tibia $\left(0^{\circ}\right.$ at the ankle joint). The angle of the ankle joint and the torque produced by contraction of the triceps surae were monitored continuously.

Vibration was applied to the Achilles tendon using a modified pneumatic drill to which an eccentric weight had been attached. The size of the weight was constant for all experiments and produced an amplitude of vibration of approximately $1.5 \mathrm{~mm}$ peak to peak. Frequency of vibration was varied from $25 \mathrm{~Hz}$ to more than $200 \mathrm{~Hz}$ by opening or closing a valve which regulated the flow of compressed air. Vibration frequency was derived from the waveform monitored using an Endevco 2222A accelerometer which was secured to the vibrator.

For studies of the tonic contraction induced by low intensity tetanic stimulation, square wave pulses of duration $1 \mathrm{~ms}$ were delivered by a Grass S-88 stimulator and stimulus isolation unit through a pair of insulated tungsten needle electrodes with bare tips of $3 \mathrm{~mm}$. The electrodes were inserted $1 \mathrm{~cm}$ apart transversely into the popliteal fossa close to the tibial nerve and were adjusted until a satisfactory $\mathrm{H}$ wave was recorded in triceps surae in response to single shocks. The EMG of tibialis anterior was monitored during adjustments of the electrode position to ensure that stimuli were delivered exclusively to the tibial nerve. In two experiments stimuli of $0.1 \mathrm{~ms}$ duration supraliminal for the $\mathbf{M}$ wave were delivered at intervals throughout the experiment to check that the stimulating needles had not been displaced.

The potentials of single motor units were recorded from the lateral gastrocnemius or soleus muscle using a needle electrode with a $25 \mu \mathrm{m}$ silver leading-off surface (Medelec), designed for single fibre EMG studies (Ekstedt, 1964; Ekstedt and Stålberg, 1973; Stålberg and Ekstedt, 1973). In these recordings the activity of single motor units was readily recorded as the potentials from 
one to three single muscle fibres. The criteria of a single muscle fibre (Ekstedt, 1964; Ekstedt and Stålberg, 1973) were: constant shape on successive discharge; rise time faster than $300 \mu \mathrm{s}$; duration less than $1000 \mu \mathrm{s}$; and amplitude greater than $200 \mu \mathrm{V}$. Commonly, vibration or voluntary contraction activated more than one motor unit within the uptake area of the electrode (approximate diameter $270 \mu \mathrm{m}$ ), but these could be differentiated on amplitude differences, the closest fibre having the largest amplitude. In addition, gross EMG activity of the lateral gastrocnemius and soleus muscles was monitored with a pair of insulated tungsten needle electrodes with bared tips of $3 \mathrm{~mm}$. These electrodes were inserted $5 \mathrm{~cm}$ apart subcutaneously or intramuscularly. Their selectivity was such that the activity of a number of motor units was usually recorded, but at times the trace was dominated by, or consisted solely of, the potentials from a single motor unit.

Data were monitored on an oscilloscope during the experiment, and recorded on an 8-channel Precision Instruments PI-6200 tape-recorder for subsequent analysis. Histograms of interdischarge intervals and of the temporal relationship of EMG spikes to the vibration wave were constructed using an Ortec 4620/4621 time histogram analyzer with 128 memory bins. Histograms of the variability of motor unit discharge relative to the vibration wave were constructed by either triggering the oscilloscope sweep with each vibration wave and recording the latency of the potential or vice versa. Similar analysis procedures were used for the potentials activated during low threshold tetanic stimulation of muscle afferent fibres. Motor unit discharge frequency was calculated from either the interdischarge interval histogram or the readout of an instantaneous frequency meter (cf. Hagbarth et al., 1975). Additionally, motor unit discharge parameters such as interdischarge variability, discharge frequency and interval histograms were computed 'off-line' using a Hewlett Packard 5326B time interval counter and PDP-40 computer.

\section{RESULTS}

MUSCLE VIBRATION The activity of 46 motor units has been studied during muscle vibration. When the triceps surae was relaxed a TVR could not be recorded in one subject despite muscle stretch and reinforcement. In the other subjects the vibration-activated motor unit potentials were clearly phase-locked to the vibration cycle. This relationship was best demonstrated by multiple superimpositions of the oscilloscope sweep, triggering the sweep from the vibration wave (Fig. 1A). Motor unit discharge frequency was usually in the range $5.5-10 \mathrm{~Hz}$, so that with vibration of $100 \mathrm{~Hz}$ most potentials occurred at intervals corresponding to 10-18 vibration cycles (Fig. 1B). Using the terminology of Homma et al. (1972a), the decoding ratios at $100 \mathrm{~Hz}$ were between $10: 1$ and $18: 1$. Sometimes the size of the decoding ratio for consecutive spikes varied apparently randomly, although with prolonged vibration a slight decrease in the ratios occurred. Construction of interdischarge interval histograms, as used by Homma and colleagues, showed a group of latencies with multiple peaks (Fig. 2A). Each peak corresponds to an integer multiple of the duration of the vibration cycle, and each major peak is separated from the next by the duration of the vibration cycle.

Synchronization of motor unit activity was seen over the entire frequency range possible with the vibrator, from the lower limit of $25 \mathrm{~Hz}$ to the upper limit of approximately $220 \mathrm{~Hz}$ (Figs 3 and 4). Motor unit firing rate showed no consistent change with change in vibration frequency-at times a slight decrease was recorded at the higher vibration frequencies, although more usually a slight increase $(0.5-1 \mathrm{~Hz})$ occurred. Thus, with higher frequencies, more vibration cycles elapsed before the next motor unit potential was generated, so that the decoding ratios were generally higher. One subject developed clonus at vibration frequencies between $100 \mathrm{~Hz}$ and $200 \mathrm{~Hz}$. Motor units firing in the clonic burst remained locked to the vibration cycle at these vibration frequencies (Fig. 4-right), although the clonus frequency varied only between $6.0 \mathrm{~Hz}$ (with vibration at $100 \mathrm{~Hz}$ ) and $6.5 \mathrm{~Hz}$ (with vibration at $200 \mathrm{~Hz}$ ).

Figure $1 \mathrm{~A}$ and $\mathrm{B}$ shows that, while motor unit potentials were locked to the vibration cycle, there was considerable variability of the phase of the cycle at which the potentials occurred. The latency histograms of these two motor units relative to their vibration cycles are shown in Fig. 2B. The degree of variability 
A
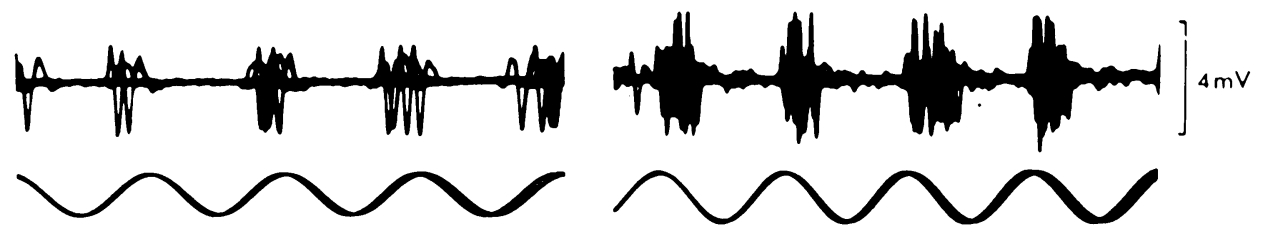

B
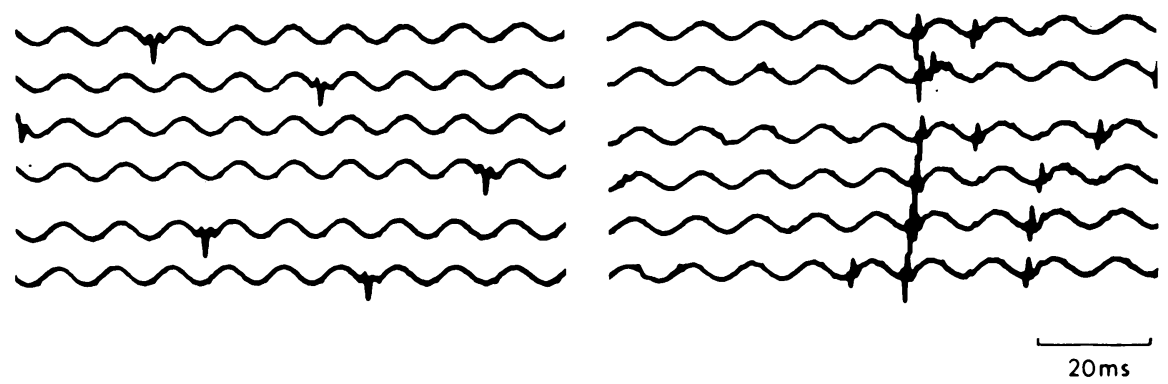

FIG. 1 Vibration-activated motor units. A. Superimposition of multiple (50-100) consecutive oscilloscope sweeps triggering from the vibration wave. Two examples; different subjects. Vibration at $80 \mathrm{~Hz}$ (left) and $90 \mathrm{~Hz}$ (right). On the right two motor units are visible, both being locked to the vibration wave. B. Successive sweeps displaced downwards with the potentials of $A$ superimposed on their vibration waves. The potentials do not occur with every vibration cycle. The potentials on the right are asynchronous with one another, even though each is locked to the vibration wave. Note that the process of preparation of these illustrations has resulted in inversion of the vibration wave, so the potentials appear to occur during the opposite phase of the cycle to that seen in $A$.

changed little with frequency (compare Fig. $2 B$ with Figs 3 and 4). At low frequencies clear gaps were visible between successive bursts of EMG activity, even after multiple superimpositions, but as frequency rose the clear intervals became briefer until at frequencies over $150 \mathrm{~Hz}$ the clusters of potentials began to merge (Fig. 3). The range of variability of latency could be determined accurately only for low frequencies of vibration and was 4-7 ms. The standard deviation of the variability (the 'jitter') as determined by computer analysis was $0.92-1.31 \mathrm{~ms}$.

With continued vibration at constant frequency a modest increase in motor unit dis- charge frequency and decrease in the decoding ratios were recorded, and the variability increased as potentials not clearly related to the vibration cycle began to appear. Additional motor units were commonly recruited within the uptake area of the electrode. The changes of discharge frequency were less than $1.0 \mathrm{~Hz}$ and the integers of the decoding ratios decreased by only 1 or 2 . Passive stretch of the triceps surae by up to $5^{\circ}$ during continued vibration resulted in recruitment of fresh motor units, and for the original units a slight decrease in the decoding ratios and no obvious change in variability. More detailed analysis of these changes was not possible as stretch 

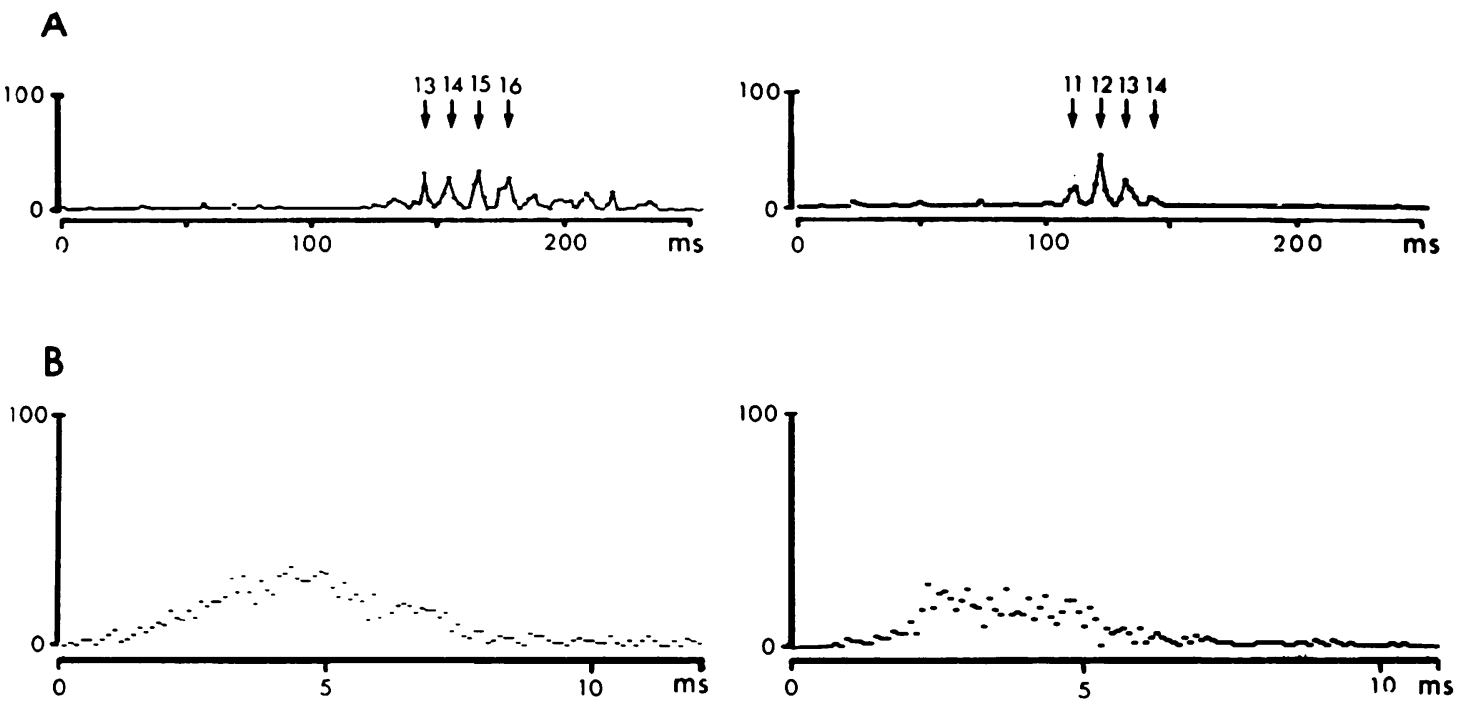

FIG. 2 Latency histograms. A. Non-sequential interdischarge interval histograms of the unit of Fig. 1 (left) and the larger unit of Fig. 1 (right). The intervals between successive discharges correspond to overall frequencies of approximately 6-8 $\mathrm{Hz}$, but in each case the histogram contains multiple peaks which represent integer multiples of the duration of the vibration cycle. The major peaks are indicated by arrows and the numbers represent the integers by which the duration of the vibration cycle can be multiplied to obtain the latency of the appropriate peak. Thus, the major decoding ratios are $13: 1$, $14: 1,15: 1$, and $16: 1$ on the left and $11: 1,12: 1,13: 1,41: 1$ on the right. The values on the y-axis represent the absolute number of discharges counted. B. Variability of motor unit potential relative to the vibration wave for the same two units as in Fig. 1 and Fig. 2 A. For technical reasons, these histograms have been obtained by triggering the averaging sweep from the potential and measuring the latency to a fixed point on the next vibration wave rather than vice versa. The duration of each histogram has been adjusted to be exactly that of one vibration cycle.

often dislodged the rigid electrode and the freshly recruited units obscured the original potentials. Abrupt return to a less stretched position resulted in abrupt cessation of EMG activity for 5-10 s even if the foot had not been returned to the original zero position.

The effects of vibration on voluntarily activated motor units were studied on 14 motor units. Motor unit potentials became recognizably locked to the vibration wave within 2-5 s of application (Fig. 5). Qualitatively similar changes were seen at all frequencies studied up to $200 \mathrm{~Hz}$. Vibration commonly increased motor unit discharge frequency slightly but this effect was not marked, the most obvious change being the regularization of the pattern of discharge. After the application of vibration, three new readily identifiable units were recruited within the uptake area of the electrode in different experiments. These units responded in a manner similar to that of the other 14 motor units. It must be emphasized that only weak voluntary contractions were used since in stronger contractions more units were activated within the uptake area, and the rigid single fibre electrode was more easily dislodged.

ELECTRICAL STIMULATION In four subjects potentials from 21 single motor units were recorded during low threshold tetanic stimulation of muscle afferents at frequencies of 25-100 Hz. Stimulus levels were subthreshold for both $\mathrm{H}$ and $\mathrm{M}$ waves (two subjects) and subthreshold for the $M$ wave but suprathreshold for the $\mathrm{H}$ wave (two subjects). With the latter form of stimulation an $\mathrm{H}$ reflex was 


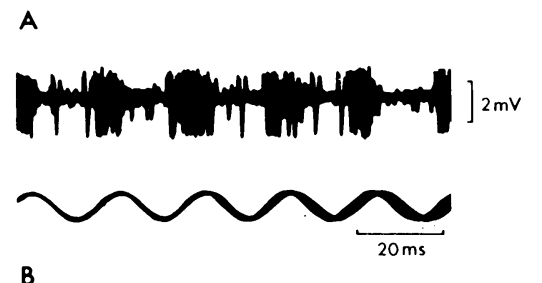

B
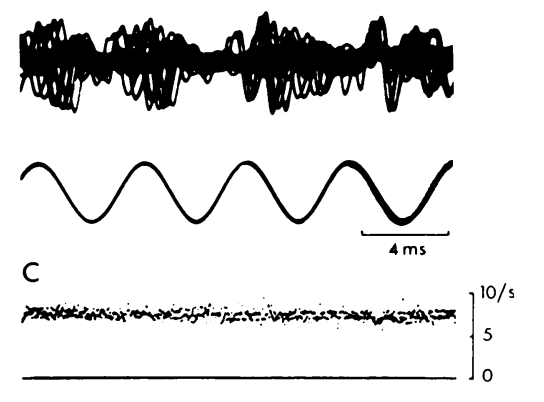

FIG. 3 Effects of changing frequency. With multiple superimpositions, as in Fig. $1 A$, a potential is clearly related to the vibration wave at 50 $\mathrm{Hz}(\mathrm{A})$ and at $220 \mathrm{~Hz}(B)$. In $\mathrm{C}$ the instantaneous frequency of the same unit decreases only slightly as vibration frequency (indicated by the accelerometer output) is decreased from $125 \mathrm{~Hz}$ to $70 \mathrm{~Hz}$.

elicited by only the first one to two stimuli. A silent period of up to $10 \mathrm{~s}$ then ensued before changes in torque and EMG activity due to the tonic contraction could be recorded (Fig. $6 \mathrm{~A}, \mathrm{~B})$.

Discharge frequency of units activated by tetanic stimulation was $5-9 \mathrm{~Hz}$. In none could convincing evidence of synchronization to the electrical stimulus be demonstrated using the same analysis techniques as used for vibration (Fig. 6C, D). These findings thus confirm those of de Gail et al. (1966) and Lang and Vallbo (1967). On the left of Fig. 7, the discharge pattern and latency histogram from a motor unit activated by tetanic stimulation shows an absence of correlation with the electrical stimulus. The same unit was then activated by vibration and can be seen on the right of Fig. 7 to be synchronized to the vibration cycle. Low threshold tetanic stimulation also failed to regularize the firing pattern of voluntarily activated motor units. No differences in behaviour were noted at the different stimulus frequencies.

In three subjects the stimulus level was raised during the course of stimulation until just suprathreshold for the $M$ response, thus producing a low-grade tetanic contraction of the muscle which was painful. The $\mathrm{M}$ response could be readily identified as a potential of fixed latency but the pattern of discharge of reflexly activated motor units did not alter, even though the higher stimulus level would have activated more afferent fibres.

\section{DISCUSSION}

EFFECTS OF VIBRATION During the TVR, motor unit firing rate remains low and, as reported previously by Lance et al. (1966) and Delwaide (1971), is not greatly affected by changing the vibration frequency. However, as maintained by Homma and colleagues, the discharge pattern is clearly related to the vibration cycle. That such a relationship could be demonstrated when the duration of the vibration cycle was as short as $5 \mathrm{~ms}$ implies that, although continuous afferent bombardment of the motoneurone might be expected at this vibration frequency, the input 
A
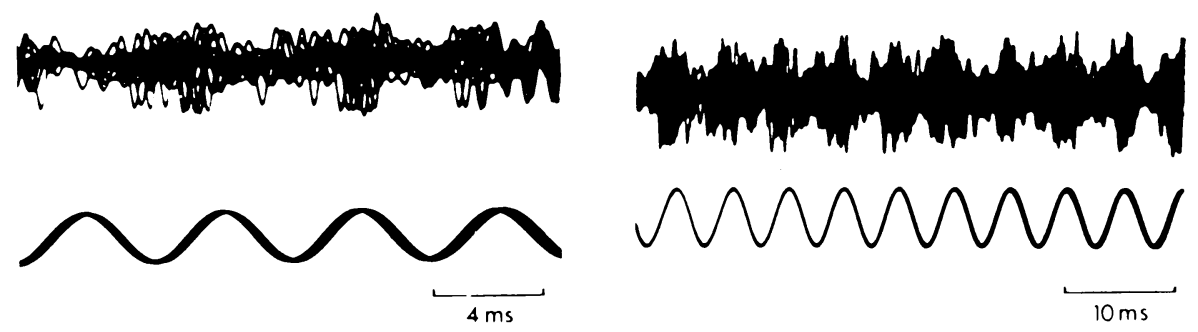

B
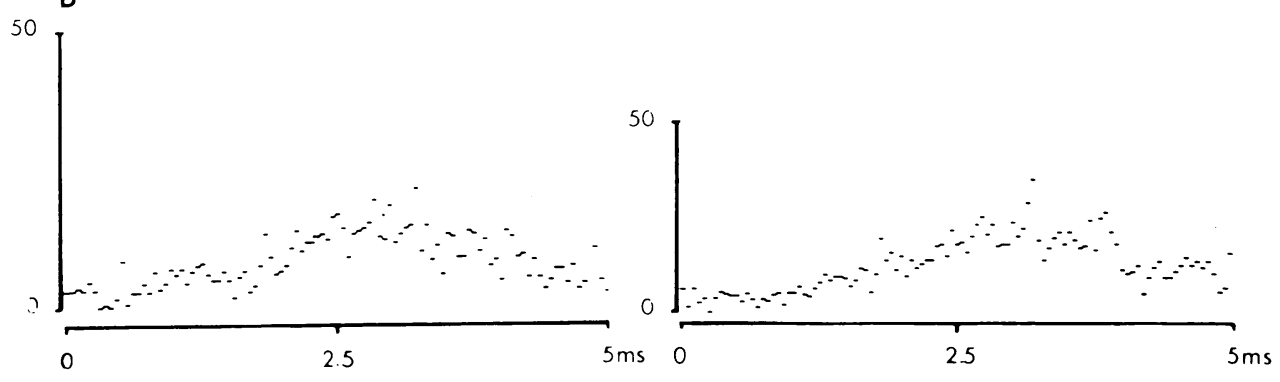

IIG. 4 Potentials activated during high frequency vibration: two examples, different subjects. A. On the left, superimpositions as in Fig. IA of discharges from predominantly one motor unit during vibration at $200 \mathrm{~Hz}$. On the right, the bursts of EMG activity contain more than one motor unit, but are clearly related to the vibration wave $(195 \mathrm{~Hz})$. This subject developed clonus at vibration frequencies between $100 \mathrm{~Hz}$ and $200 \mathrm{~Hz}$, and when superimposed as in Figure on the right, EMG potentials recorded in the clonic bursts were related to the vibration cycle at all frequencies. B. Histograms of the variability of motor unit discharge relative to vibration wave as in Fig. $2 B$ for the unit of $A$-left and the largest unit in the bursts of A-right. Note that the histogram durations have again been adjusted for these short vibration cycles. Motor unit discharge occurs throughout the entire vibration cycle, but the absolute variability is of the same magnitude as in Fig. $2 B$ where the vibration cycle is twice as long.

responsible for the timing of motor unit discharge must retain a pulsatile quality. The presence of a demonstrable relationship between vibration wave and motoneurone response necessitates rapid and direct reflex transmission and is therefore suggestive of a monosynaptic pathway (Homma and Kanda, 1973). With the higher frequencies and longer reflex arc used in the present study, the interpolation of an interneuronal chain could only further desynchronize the already somewhat dispersed afferent volley. Thus, it seems reasonable to conclude that under the present experimental conditions the vibration-induced triggering of motor discharges is largely the result of activity in the monosynaptic arc.

Although clearly locked to the vibration cycle, the timing of motoneurone firing varied by up to $7 \mathrm{~ms}$, the standard deviation of variability (the 'jitter') being of the order of 1.0 $1.3 \mathrm{~ms}$. By contrast, the range of variability for $90 \%$ of motoneurones activated in the $\mathrm{H}$ reflex has been reported to be $0.4-2 \mathrm{~ms}$, with a standard deviation of $200 \mu \mathrm{s}$ (Trontelj, 1973). The discrepancy between the values for the $\mathrm{H}$ reflex and vibration-activated motor units is high. Presumably most if not all the difference may be attributed to the higher degree of syn- 
A

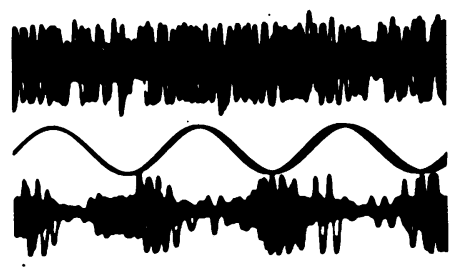

C

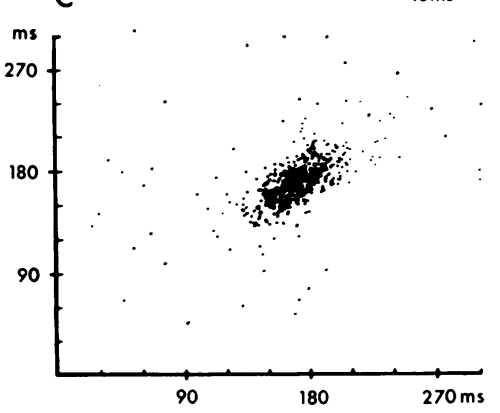

B

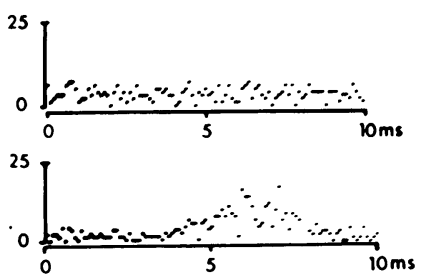

D

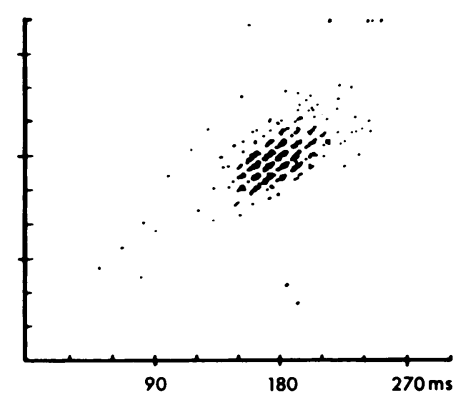

FIG. 5 Vibration and voluntarily activated motor units. A. Upper trace-voluntarily activated unit discharging in a fashion unrelated to the vibration wave (middle trace) which triggers the sweep although not yet applied to the muscle. The slight differences in amplitude are due to movement of the recording electrode. Lower trace-same motor unit, still under voluntary control, but with vibration applied. Two motor units can be seen, both locked to the vibration wave. B. Histograms of variability of discharge of the larger potential in $A$ relative to vibration wave. Upper histogram-vibration not applied, showing as expected no relationship between discharge and vibration wave, Lower histogram-vibration applied. Voluntary activity now synchronized to the vibration. C. Joint interval histogram, showing each interdischarge interval plotted against the next interdischarge interval, for a different voluntarily-activated motor unit from that in $A$ and $B$. Vibration not yet applied. Overall discharge frequency varies between 5-7 Hz. D. Same motor unit as in C, same type of display, but with vibration applied. Discharge frequency remains much the same overall. The intervals between successive discharges are now multiples of the duration of the vibration cycle so that the plot has become organized into lines which are separated horizontally and vertically from each other by an interval equal to the duration of the vibration cycle.

chrony in the electrically-activated Ia volleys, as compared with those activated mechanically by vibration, and there is no need to postulate involvement of an oligo- or polysynaptic circuit to account for the high variability. However, the changes in motoneurone response that occur with continued vibration - the decrease in decoding ratios and the tendency for discharges to appear unrelated to the vibration cycle-suggest the presence of an additional pathway for processing afferent information over and above that which is responsible for the vibration-induced timing of motor unit discharge. Based on intracellular recordings from feline gastrocnemius motoneurones made by Homma and Kanda (1973), these changes in motoneurone firing pattern in man have been attributed to the development of a steady background excitatory postsynaptic potential (EPSP) due to poly- 
A

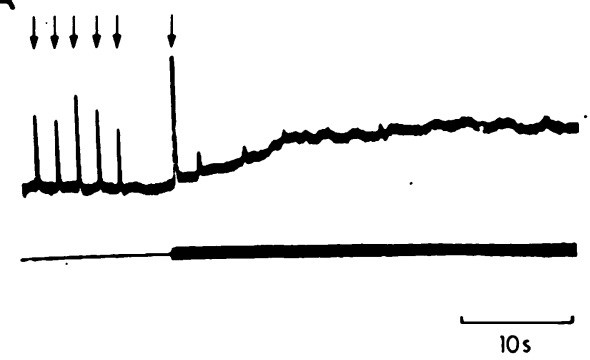

C

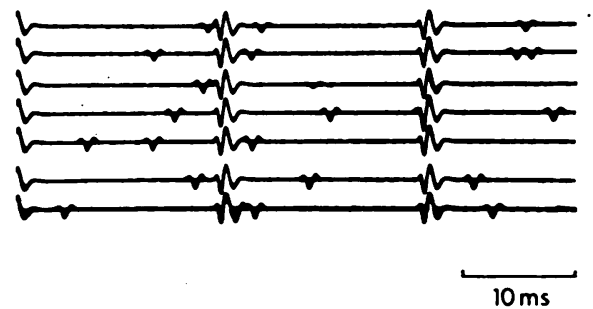

B

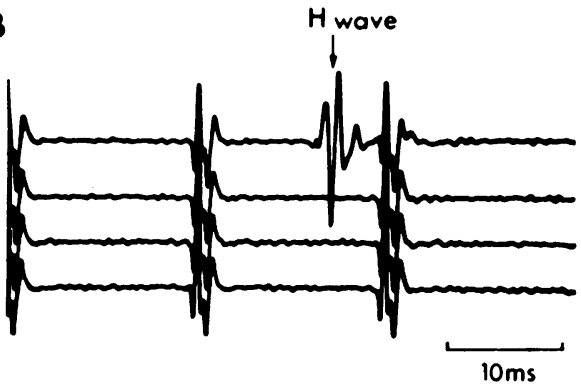

D

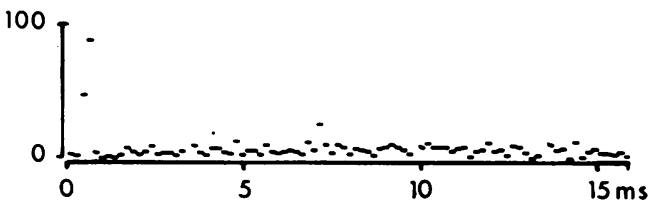

FIG. 6 Tetanic stimulation of muscle afferents at $60 \mathrm{~Hz}$. A. Torque changes (upper trace) induced by five control $H$ reflexes (arrowed) at 1 per $2 s$ and, after a $5 s$ pause, tetanic stimulation at $60 \mathrm{~Hz}$ at the same stimulus level. The $\mathrm{H}$ reflex evoked by the first stimulus of the train (arrowed) is larger than the controls due to the 5 s pause. A slowly increasing tonic contraction then develops. The thickening of the lower trace indicates tetanic stimulation. B. The EMG of the first 12 stimuli of the same tetanic train contains only 12 stimulus artefacts and one EMG potential (arrowed) occurring $28 \mathrm{~ms}$ after the first stimulus and representing the $\mathrm{H}$ reflex response to this first stimulus. There is no visible $H$ reflex response to subsequent stimuli. Stimulation was below $M$ wave threshold. $C$. Later in the stimulus train. A single motor unit is recorded, discharging in a fashion unrelated to the stimuli. The oscilloscope was triggered so that, after superimposing nine consecutive sweeps (representing a total of 27 stimuli), the oscilloscope beam stepped down to create the next trace. Thus, the seven traces represent $27 \times 7$ consecutive stimuli. A total of 21 motor unit discharges are seen in this time, representing a discharge frequency of $6.7 \mathrm{~Hz}$. D. Histogram (as in Fig. $2 \mathrm{~B}$ ) of the variability of discharge of the potential in $C$ relative to the electrical stimulus. Discharge appears unrelated to the stimulus.

synaptic transmission (Hirayama et al., 1974). As described by Homma and colleagues, the characteristics of the human TVR may be explained by a slowly developing steady excitatory state caused by transmission in polysynaptic pathways, upon which are superimposed vibration-synchronous EPSPs due to transmission in the monosynaptic pathway, motoneurone spikes being generated when the total depolarization exceeds threshold.

The extent of the latency 'jitter' for single motor units activated in the TVR of triceps surae appears to be similar to that reported by Godaux et al. (1975) for the TVR of limb muscles, but is significantly greater than the 'jitter' of single motor units activated in the tendon jerks of the same muscles (Godaux $e t$ al., 1975). This difference has led Godaux et al., 1975) to conclude that the monosynaptic pathway contributes little to the timing of motor discharge in the TVR of limb muscles, but the evidence for this conclusion can be 
A
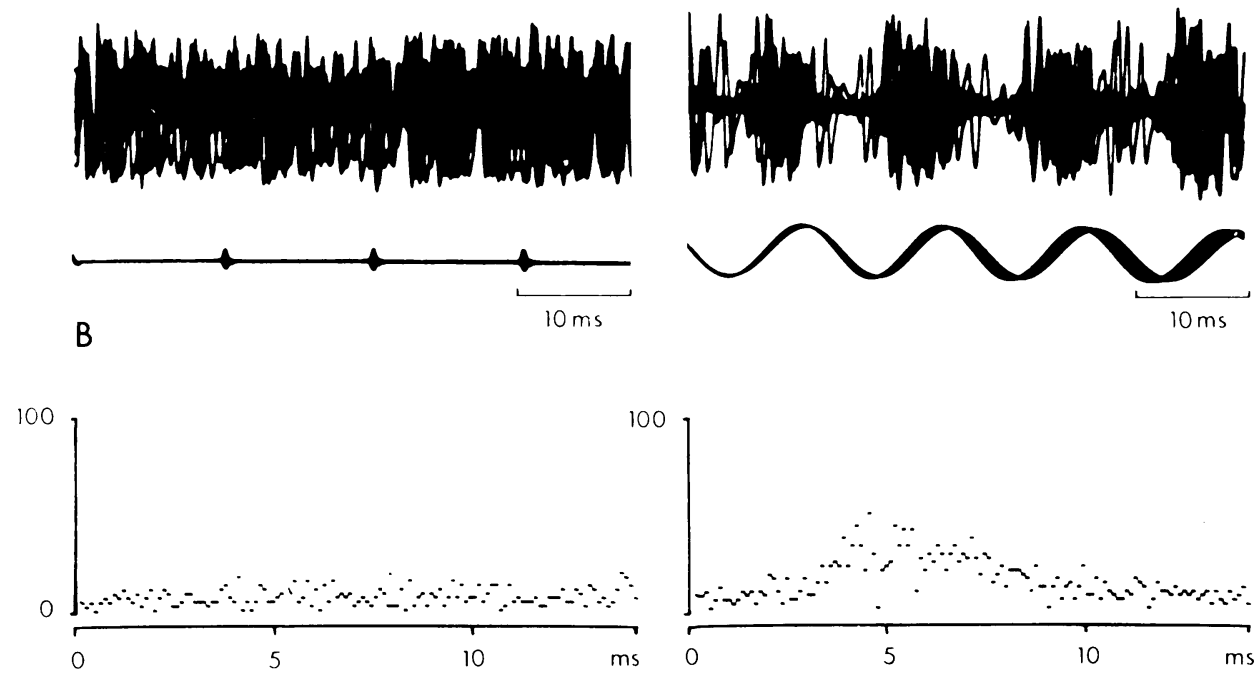

III. 7 Activation of a motor unit by tetanic stimulation and by vibration. A. On the left multiple superimpositions of a large motor unit potential activated by tetanic stimulation (approximately 70 discharges). Discharge is apparently unrelated to the electrical stimulus (lower trace) which is used to trigger the oscilloscope sweeps. On the right, vibration at the same frequency $(70 \mathrm{~Hz})$ activates the same unit (and another unit, represented by a smaller potential). Discharge is locked to the vibration wave. B. Histograms, as in Fig. 2B, of the variability of discharge of the motor unit of $A$ when activated by tetanic stimulation (left) and by vibration (right).

debated. Firstly, the tendon jerk is generated by a single mechanical stimulus which presumably evokes a large amplitude EPSP with a fast rise time, while the less intense vibratory stimulus would produce a lower amplitude EPSP with a slower rise time. Additionally, the propagation velocity of the vibration wave is such that spindles at different ends of large muscles are activated at different phases of the cycle, an afferent dispersion which would be accentuated by the long reflex arc. Furthermore, the slightly delayed input from tendon organs and secondary endings would become superimposed on the monosynaptic depolarization during a sequence of vibration cycles. Such factors would be more important the larger the muscle and the longer the reflex arc, but their effects would be less obvious in a small muscle with a very short reflex arc, such as the masseter. Indeed, Godaux and Desmedt (1975) have reported the 'jitter' of EMG spikes in the jaw jerk to be similar to that of the masseter TVR.

EFFECTS OF ELECTRICAL STIMULATION At first sight the failure of motoneurones activated during low threshold tetanic stimulation to be time-locked to the electrical stimulus seems difficult to reconcile with the pattern of vibration-activated motor units. Certainly, electrical stimulation should produce a less dispersed input than vibration since the uncertainties of the spindle transducing mechanisms and the distal afferent fibres are bypassed. When stimulating at a level supraliminal for the $H$ reflex but subthreshold for the $M$ wave, the $H$ reflex was elicited by only the first couple of stimuli, the subsequent failure of response indicating 
suppression of transmission in the monosynaptic pathway. After the ensuing pause, the potentials which appeared were not obviously related to the stimulus and were presumably generated through a polysynaptic circuit.

Irrespective of the reasons for the asynchrony of a motor unit discharge during low threshold tetanic stimulation, the lack of synchrony does provide some indication of the relative importance of monosynaptic and polysynaptic pathways in generating a TVR. The development of a tonic contraction solely on the basis of polysynaptic activity during the electrical stimulation suggests that, in isolation, the polysynaptic pathway is an adequate pathway. On the other hand the TVR in normal man may not appear until a few seconds after the application of vibration, a fact indicating that, in isolation, the monosynaptic pathway is incapable of producing a tonic contraction.

WORKING HYPOTHESIS AND ITS IMPLICATIONS While alternative mechanisms cannot be excluded, a reasonably satisfying working hypothesis can be advanced to explain the differences in motoneurone discharge pattern in the vibration-induced and electricallyinduced contractions, if it is assumed: (a) that the vibration-induced timing phenomenon is the result of activity in predominantly the monosynaptic pathway, as discussed above; (b) that the electrical stimulus is a less selective afferent fibre stimulus than vibration, so that the stimulus activated a greater proportion of $\mathrm{Ib}$ fibres than did vibration, and possibly may have activated group II or even cutaneous afferent fibres as well.

The suppression of the $\mathrm{H}$ wave lasted throughout the stimulus train, was complete many seconds before the asynchronous tonic discharges began, and therefore cannot be readily explained by recurrent inhibitory effects from Renshaw cell activation. Postsynaptic inhibition from Ib (and possibly other) afferent fibres certainly would have occurred, presumably for the duration of the stimulus train, but was probably not the sole inhibitory factor operating, as with a purely postsynaptic inhibitory mechanism, the direct monosynaptic pathway should remain the pathway of lowest threshold. The development of asynchronous tonic discharges therefore could imply that the monosynaptic pathway was also inhibited in its afferent course. Presynaptic inhibition of the extensor monosynaptic pathway due to conditioning activity in different primary afferent fibres is well recognized in the cat (cf. Barnes and Pompeiano, 1970, for references) and possibly also in man ( $c f$. Delwaide, 1971, 1973), and could be a factor in the failure of monosynaptic transmission.

In response to low threshold tetanic stimulation of the tibial nerve it seems likely, therefore, that the tonic contraction is generated by spindle afferent activity through polysynaptic circuits, that the monosynaptic pathway is suppressed at both pre- and postsynaptic levels, and that the level of activity reaching the motoneurone through the suppressed monosynaptic pathway is insufficient to synchronize motoneurone firing pattern. Presynaptic inhibition may be induced by vibration (Gillies, et al., 1969; Barnes and Pompeiano, 1970; Delwaide, 1971, 1973; Thoden et al., 1972), so that basically similar circuits are probably activated during muscle vibration. The apparently paradoxical ability of vibration to impose a vibration-related pattern of motoneurone discharge when electrical stimulation failed to do so may then be explained by two factors: a lower level of suppression of the monosynaptic pathway due to more selective group Ia activation; and a higher level of group Ia afferent activity induced by the powerful vibratory stimulus compared with the necessarily low intensity electrical stimulus so that greater excitation reached the motoneurone through the presynaptic 'gate'.

This hypothesis receives some support from recently published experiments by Homma et al. (1975) who studied the effects produced by repetitive electrical stimulation at $16-100 \mathrm{~Hz}$ of the nerve to the gastrocnemii in chloralose/ urethane anaesthetized cats. Stimulation at a level 'just above the threshold of group I fibers' produced reflex effects and intracellular 
potential changes similar to those previously obtained with vibration by Homma and colleagues in cat and man, and in the present study in man. Precise values for this level of stimulation were not given, but in this nerve in the cat it is likely that stimuli 'just above the threshold of group I fibers' elicited activity in group I fibres of only spindle origin (Coppin et al., 1969, 1970). At a higher stimulus intensity (5.0 times group I threshold), Homma et al. (1975) found a decrease in amplitude of the monosynaptic EPSP ripple which they attributed to the activation of group II afferent fibres, although their experiments did not exclude a contribution from group Ib afferent fibres. The present experiments in man are probably analogous to the less-selective higher stimulus intensity used by Homma et al. (1975).

Thus, these studies are compatible with the operation of two reflex pathways during muscle vibration, the monosynaptic pathway which may be subjected to inhibitory modulation, and a polysynaptic pathway which is not so modulated (Kanda, 1972; Hirayama, et al., 1974). The results support the suggestion of Hagbarth et al. (1976) that the monosynaptic pathway plays little or no role in the generation of the TVR, whereas it plays an essential role in the vibration-induced timing of motoneurone discharge. However, in pathological states such as spasticity, the monosynaptic pathway could be of greater significance in generating the tonic contraction. Based on indirect evidence, it has been suggested that presynaptic inhibitory mechanisms are suppressed in spasticity (Delwaide, 1971, 1973; Burke and Ashby, 1972; Ashby et al., 1974). The absence of such modulation of the monosynaptic pathway could be a factor in the abrupt onset of the TVR which is characteristic of spasticity (Hagbarth and Eklund, 1968; Burke et al., 1972).

The authors are grateful to Professor Karl-Erik Hagbarth and Dr Erik Stålberg for helpful discussion and use of facilities.

\section{REFERENCES}

Ashby, P., Verrier, M., and Lightfoot, E. (1974). Segmental reflex pathways in spinal shock and spinal spasticity in man. Journal of Neurology, Neurosurgery, and Psychiatry, 37, 1352-1360.

Barnes, C. D., and Pompeiano, O. (1970). Presynaptic and postsynaptic effects in the monosynaptic reflex pathways to extensor mononeurones following vibration of synergic muscles. Archives of Italian Biology, 108, 259-294.

Burke, D., Andrews, C. J., and Lance, J. W. (1972). Tonic vibration reflex in spasticity, Parkinson's disease, and normal subjects. Journal of Neurology, Neurosurgery, and Psychiatry, 35, 477-486.

Burke, D., and Ashby, P. (1972). Are spinal 'presynaptic' inhibitory mechanisms suppressed in spasticity? Journal of the Neurological Sciences, 15, 321-326.

Coppin, C. M. L., Jack, J. J. B., and McIntyre, A. K. (1969). Properties of group Ia afferent fibres from semitendinosus muscle in the cat. Journal of Physiology, 203, 45-46P.

Coppin, C. M. L., Jack, J. J. B., and MacLennan, C. R. M. (1970). A method for selective electrical activation of tendon organ afferent fibres from the cat soleus. Journal of Physiology, 210, 18-20P.

de Gail, P., Lance, J. W., and Neilson, P. D. (1966). Differential effects on tcnic and phasic reflex mechanisms produced by vibration of muscles in man. Journal of Neurology, Neurosurgery, and Psychiatry, 29, 1-11.

Delwaide, P. J. (1971). Étude Expérimentale de l'Hyperréflexie Tendineuse en Clinique Neurologique. Editions Arscia: Brussels.

Delwaide, P. J. (1973). Human monosynaptic reflexes and presynaptic inhibition. In New Developments in EMG and Clinical Neurophysiology, vol. 3, pp. 508-522. Edited by J. E. Desmedt. Karger: Basel.

Desmedt, J. E., and Godaux, E. (1975). Vibrationinduced discharge patterns of single motor units in the masseter muscle in man. Journal of Physiology, 253, 429-442.

Ekstedt, J. (1964). Human single muscle fibre action potentials. Acta Physiologica Scandinavica, 61, suppl. 226, 1-96.

Ekstedt, J., and Stålberg, E. (1973). Single fibre electromyography for the study of the microphysiology of the human muscle. In $\mathrm{New}$ Developments in EMG and Clinical Neurophysiology, vol. 1, pp. 89-112. Edited by J. E. Desmedt. Karger: Basel.

Gillies, J. D., Lance, J. W., Neilson, P. D., and Tassinari, C. A. (1969). Presynaptic inhibition of the monosynaptic reflex by vibration. Journal of Physiology, 205, 329-339.

Godaux, E., and Desmedt, J. E. (1975). Evidence for a monosynaptic mechanism in the tonic vibration reflex of the human masseter muscle. Journal of Neurology, Neurosurgery, and Psychiatry, 38, 161-168. 
Godaux, E., Desmedt, J. E., and Demaret, P. (1975). Vibration of human limb muscles: the alleged phase-locking of motor unit spikes. Brain Research, 100, 175-177.

Hagbarth, K-E., and Eklund, G. (1968). The effects of muscle vibration in spasticity, rigidity, and cerebellar disorders. Journal of Neurology, Neurosurgery, and Psychiatry, 31, 207-213.

Hagbarth, K-E., Hellsing, G., and Löfstedt, L. (1976). TVR and vibration-induced timing of motor impulses in the human jaw elevator muscles. Journal of Neurology, Neurosurgery, and Psychiatry, 39, 719-728.

Hagbarth, K-E., Wallin, G., and Löfstedt, L. (1975). Muscle spindle activity in man during voluntary fast alternating movements. Journal of Neurology, Neurosurgery, and Psychiatry, 38, 625-635.

Hirayama, K., Homma, S., Mizote, M., Nakajima, Y., and Watanabe, S. (1974). Separation of the contributions of voluntary and vibratory activation of motor units in man by cross-correlograms. Japanese Journal of Physiology, 24, 293-304.

Homma, S., and Kanda, K. (1973). Impulse decoding process in stretch reflex. In Motor Control, pp. 45-64. Edited by A. A. Gydikov, N. T. Tankov, and D. S. Kosarov. Plenum: New York.

Homma, S., Kanda, K., and Watanabe, S. (1972a). Preferred spike intervals in the vibration reflex. Japanese Journal of Physiology, 22, 421-432.

Homma, S., Kanda, K., and Watanabe, S. (1972b). Integral pattern of coding during tonic vibration reflex. In Neurophysiology Studied in Man, pp. 345-349. Edited by G. G. Somjen. Excerpta Medica: Amsterdam.

Homma, S., Mizote, M., and Watanabe, S. (1975). Participation of mono- and polysynaptic transmission during tonic activation of the stretch reflex arcs. Japanese Journal of Physiology, 25, 135-146.

Kanda, K. (1972). Contribution of polysynaptic pathways to the tonic vibration reflex. Japanese Journal of Physiology, 22, 367-377.

Lance, J. W., de Gail, P., and Neilson, P. D. (1966). Tonic and phasic spinal cord mechanisms in man. Journal of Neurology, Neurosurgery, and Psychiatry, 29, 535-544.

Lang, A. H., and Vallbo, A. B. (1967). Motoneuron activation by low intensity tetanic stimulation of muscle afferents in man. Experimental Neurology, 18, 383-391.

Stålberg, E., and Ekstedt, J. (1973). Single fibre EMG and microphysiology of the motor unit in normal and diseased human muscle. In New Developments in EMG and Clinical Neurophysiology, vol. 1, pp. 113-129. Edited by J. E. Desmedt. Karger: Basel.

Thoden, U., Margherini, P. C., and Pompeiano, O. (1972). Evidence that presynaptic inhibition may decrease the autogenetic excitation caused by vibration of extensor muscles. Archives of Italian Biology, 110, 90-116.

Trontelj, J. V. (1973). A study of the H-reflex by single fibre EMG. Journal of Neurology, Neurosurgery, and Psychiatry, 36, 951-9.59. 